Int. J. Morphol.,

32(3):850-859, 2014

\title{
EVEA en Anatomía: Adhesión y Gestión de Contenidos por Áreas
}

\author{
EVEA in Anatomy: Accession and Content Management by Area
}

\begin{abstract}
Rubén Daniel Algieri*; Elba Beatriz Tornese**; Martín Javier Mazzoglio y Nabar ${ }^{* * *}$; Claudia Gabriela Dogliotti*****; Andrea Gazzotti ${ }^{* * * * *}$; Humberto Nicolás Jiménez Villarruel ${ }^{* * * * * *} \&$ Lorena Rey $^{* * * * * * * *}$
\end{abstract} ALGIERI, R. D.; TORNESE, E. B.; MAZZOGLIO Y NABAR, M. J.; DOGLIOTTI, C. G.; GAZZOTTI, A.; JIMÉNEZ
VILLARRUEL, H. N. \& REY, L. EVEA en Anatomía: adhesión y gestión de contenidos por áreas. Int. J. Morphol., 32(3):850-859,
2014.

RESUMEN: Estudios preliminares ratificaron beneficios cuali-cuantititavos con el uso de espacios virtuales de enseñanza y aprendizaje (EVEA) en Anatomía tanto en el rendimiento cognoscitivo como en aspectos socioeducativos y pedagógicos. Objetivo: Evaluar la adhesión, gestión de contenidos y uso de alumnos de grado con un EVEA según las áreas que componen la materia y características de los usuarios. Estudio observacional, retrospectivo y transversal sobre características de uso y gestión de contenidos (administrativa y académica) en actividades de anatomía clínica (anatomo-quirúrgicas y anátomo-imagenológicas) a través de un EVEA en 309 alumnos del ciclo 2011. Se dividieron las actividades por áreas de la materia y aplicaron parámetros estadísticos (descriptivos e inferenciales) a los resultados. El trabajo cumplió con las normativas ético-legales vigentes. La mayoría ingresaba al EVEA desde espacios públicos con conexión y con computadores móviles. Las 3 áreas presentaron un promedio de 4/5 ingresos por semana, diferenciándose en que durante esplacnología los usuarios no ingresaban el mismo día del trabajo práctico cadavérico y con el mayor tiempo de permanencia. Las actividades con casos clínico-quirúrgicos presentaron mayores intentos de realización con peores resultados. Las horas laborales fueron correlativas con parámetros de uso (cantidad y tiempo de logueo). La adhesión al espacio virtual presentó especificidades según las áreas de la materia que estuvieron relacionadas con las características laborales de los usuarios e incidieron en el rendimiento obtenido en las actividades acorde con la estrategia pedagógica de las mismas.

PALABRAS CLAVE: Anatomía; Pedagogía; Espacio virtual de enseñanza y aprendizaje; Adhesión; Gestión.

\section{INTRODUCCIÓN}

En estudios preliminares determinamos los beneficios cuali y cuantitativos alcanzados por los estudiantes universitarios de grado mediados por el uso de un espacio virtual de enseñanza y aprendizaje (EVEA) diseñado con la plataforma Moodle® (Algieri et al., 2009; Algieri et al., 2011; Algieri et al., 2012a, 2012b, 2012c; Tornese et al., 2011).
Este tipo de tecnologías de la información y la comunicación (TIC) se han introducido de manera sólida en el desarrollo de las currículas de materias tanto universitarias como no universitarias de todos los niveles de la educación con especificidades y finalidades análogas. Este impacto se debe a las múltiples posibilidades de interacción bidireccional entre los usuarios (alumno-alumno y alumno-

* Médico, Especialista en Cirugía General. Profesor Adjunto de Anatomía e Histología, UM. Jefe Trabajos Prácticos de Anatomía, III Cátedra de Anatomía, Universidad de Buenos Aires, Buenos Aires, Argentina.

** Médica, Doctora en Medicina, Médica Psiquiatra y Médica Legista de la UBA. Profesora Titular de la Universidad Abierta Interamericana. Jefa de Servicio del Hospital Neuropsiquiátrico "Dr. Braulio A. Moyano”. Profesora Adjunta Equiparada de Anatomía y Docente Autorizada de Salud Mental, Facultad de Medicina, Universidad de Buenos Aires, Buenos Aires, Argentina.

*** Médico, Especialista en Psiquiatría. Docente Adscripto de la Facultad de Medicina, UBA. Jefe de Trabajos Prácticos de Anatomía, III Cátedra de Anatomía, Docente Auxiliar de Farmacología y de Psiquiatría, Facultad de Medicina, Universidad de Buenos Aires, Buenos Aires, Argentina.

***** Médica, Especialista en Psiquiatría. Docente Adscripta de la Facultad de Medicina, UBA.Jefe de Trabajos Prácticos de Anatomía, III Cátedra de Anatomía, Facultad de Medicina, Universidad de Buenos Aires, Buenos Aires, Argentina.

****** Médica, Especialista en Medicina Física y Rehabilitación Física. Docente Adscripta de la Facultad de Medicina, UBA. Jefe de Trabajos Prácticos de Anatomía, III Cátedra de Anatomía, Facultad de Medicina, Universidad de Buenos Aires, Buenos Aires, Argentina.

******* Médico, Especialista en Cirugía y en Cirugía Plástica y Reparadora, Jefe de Trabajos Prácticos de Anatomía, III Cátedra de Anatomía, Facultad de Medicina, Universidad de Buenos Aires, Buenos Aires, Argentina.

********* Médica, Especialista en Pediatría. Jefe de Trabajos Prácticos de Anatomía, III Cátedra de Anatomía, Facultad de Medicina, Universidad de Buenos Aires, Buenos Aires, Argentina. 
docente) en un contexto social de tipo horizontal que favorece el aprendizaje (Inzunza \& Bravo, 1999; Lara \& Duart, 2005; Popescu \& Navarro, 2005), en concordancia con lo postulado por Vigotsky.

En concordancia con publicaciones internacionales, nuestro grupo de investigación halló beneficios tanto objetivos (rendimiento de los alumnos asociados con el uso del EVEA, cantidad de horas de relación entre el alumno con la materia, abordaje de los temas mediante distintas estrategias pedagógicas) como subjetivos (relación del educando con sus pares, con el desarrollo de la materia y con la internalización y generación de nuevos escenarios educativos) de los EVEAS aplicados en el proceso de enseñanza y aprendizaje como una herramienta complementaria al desarrollo curricular clásico de la materia (Avila et al., 2004; Buelta Carrillo et al., 2003; Inzunza et al., 1993; Inzunza \& Bravo, 2002). Además de los beneficios descritos y las posibilidades de maximizar esquemas cognitivos por medio de su uso (Tornese et al., 2012), este tipo de TIC cumple con la particularidad de poder utilizarse las 24 horas por cualquier canal de conexión (computadora, notebook, netbook, celular, con conexiones móviles o no) (Tornese et $a l ., 2012)$ y permitir a sus usuarios imaginar y generar innovaciones dentro del mismo, haciéndolos partícipes y no meros receptores (Wiecha \& Barrie, 2005).

El objetivo del presente trabajo de investigación fue evaluar la adhesión y el uso por parte de los alumnos universitarios con un EVEA aplicado al proceso de enseñanza y aprendizaje de la Anatomía Humana, con especificación de las áreas que componen la materia. Asimismo, evaluar los parámetros relativos a la gestión de los contenidos y correlacionar los resultados en función de los datos socioeconómicos de los usuarios.

\section{MATERIAL Y MÉTODO}

Se realizó un estudio observacional, retrospectivo, de tipo transversal sobre la implementación de un EVEA diseñado con la plataforma Moodle ${ }^{\circledR}$ que fue implementado como un recurso instruccional complementario a los trabajos prácticos con material cadavérico y a las clases teóricas brindadas por docentes de la Cátedra. Se estudió la adhesión de los usuarios en las actividades de anatomía clínica que se presentaban: casos clínico-quirúrgicos, correlatos de imágenes estructurales con preparados anatómicos y casos de imagenología con reconstrucciones en 3D. También se valoró la gestión de los contenidos, tanto administrativa como académica, que fueron utilizados por los alumnos a través del espacio virtual y por los docentes para evaluar el proceso enseñanza-aprendizaje. Se valoró, según área de la materia, el promedio de veces que realizó las actividades, las necesarias para aprobar las mismas, el porcentaje de veces (intentos) aprobados y reprobados y el porcentaje de alumnos que reprobaron repetidamente 2 o más veces la misma actividad.

La población de usuarios estuvo formada por 309 alumnos de 3 comisiones del año 2011 correspondientes a la cursada de Anatomía de la $3^{\circ}$ Cátedra de Anatomía de la Facultad de Medicina de la Universidad de Buenos Aires que realizaron de forma completa todas las actividades mencionadas en sus distintos formatos. Aquellos alumnos que no habían realizado todas las actividades (por haber perdido la condición de regularidad o por falta de adherencia al presente recurso pedagógico) fueron excluidos de la presente investigación. Las características poblacionales de la muestra se exhiben en la Tabla I.

Se extrajeron de las estadísticas que provee el programa Moodle $\AA$ los resultados de los parámetros de adhesión y se valoró la gestión de los contenidos del EVEA (en tanto seguimiento y evaluación de las actividades), con especificación de las características particulares de cada área de la materia. Se obtuvo un promedio de los resultados por

Tabla I. Características poblacionales de la muestra.

\begin{tabular}{lcr}
\hline Sexo & Masculino & $31,07 \%$ \\
& Femenino & $68,93 \%$ \\
Edad & $18-24$ & $85,44 \%$ \\
& $25-30$ & $12,62 \%$ \\
Trabaja & $>30$ & $1,94 \%$ \\
& $\mathrm{Si}$ & $65,70 \%$ \\
& $\mathrm{No}$ & $34,30 \%$ \\
Horas de trabajo en la semana & Entre $12 \mathrm{y} 24 \mathrm{~h} / \mathrm{semana}$ & $44,98 \%$ \\
& Entre $25 \mathrm{y} 30 \mathrm{~h} / \mathrm{semana}$ & $35,60 \%$ \\
& Entre $30 \mathrm{y} 40 \mathrm{~h} / \mathrm{semana}$ & $13,59 \%$ \\
¿Tiene como estuadiante alguna experiencia & $>40 \mathrm{~h} / \mathrm{semana}$ & $5,83 \%$ \\
previa en el uso de e-learning? & $\mathrm{Si}$ & $7,44 \%$ \\
\hline
\end{tabular}


área (Locomotor, Esplacnología, Neuroanatomía) que fueron procesados en formato de gráfico para su mejor comprensión y análisis. Fueron excluidos los resultados obtenidos en los ingresos durante la semana de repaso previa al parcial de cada área, pues los alumnos vuelven a tener acceso a todos los TP.

Los resultados fueron sometidos a pruebas de estadística descriptiva (promedio, máximo, mínimo) e inferencial (correlación $\mathrm{r}_{\text {y }} \mathrm{r}^{2}$ ) y se realizaron los gráficos mediante la utilización del Microsoft Excel® 2007 para Windows. El presente trabajo de investigación se realizó atento a los reparos éticos y normativos vigentes (requisitos de las Good Clinical Practices -GCP-, disposiciones regulatorias y adhesión a principios éticos con origen en la Declaración de Helsinski).

\section{RESULTADOS}

Dentro de los parámetros de uso con el EVEA, el tipo de conexión para acceder al mismo fue en el $46.60 \%$ de los usuarios tanto mediante conexión móvil como no móvil. El lugar preferente para ingresar al espacio virtual fue el acceso público, seguido por los domicilios particulares, espacios laborales y por último desde la facultad. El instrumento tecnológico más utilizado para ingresar lo constituyeron las computadoras tipo notebook/netbook, seguidas por las desktop (de escritorio), y se destacó el aumento porcentual y sostenido de uso para fines educativos de los smartphone y tablets PC (Fig. 1). Determinamos en las 3 áreas de la materia un promedio de ingresos por semana prevalente (entre 4 y 5), seguido por 3 ingresos por semana. La cantidad de ingresos por día prevalente también coinci-

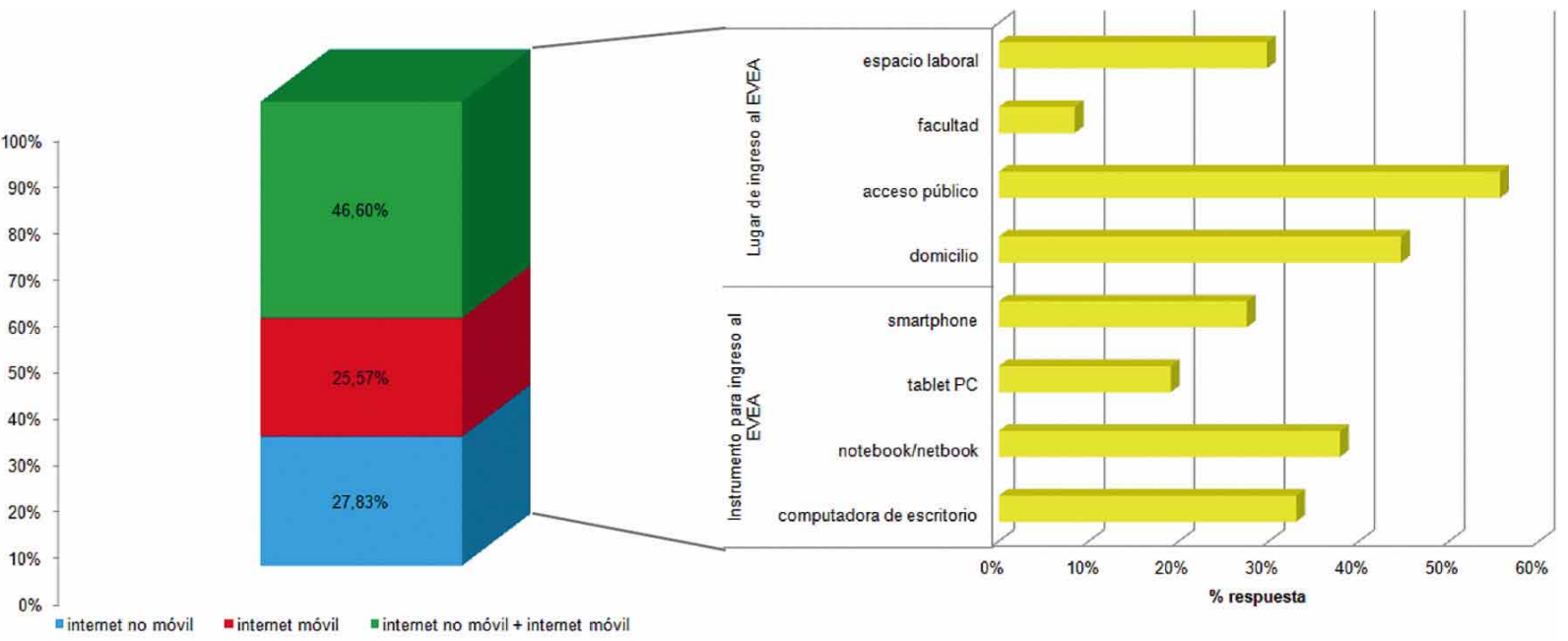

Figura 1. Medios de conexión con especificación del instrumento utilizado y lugar físico de ingreso.

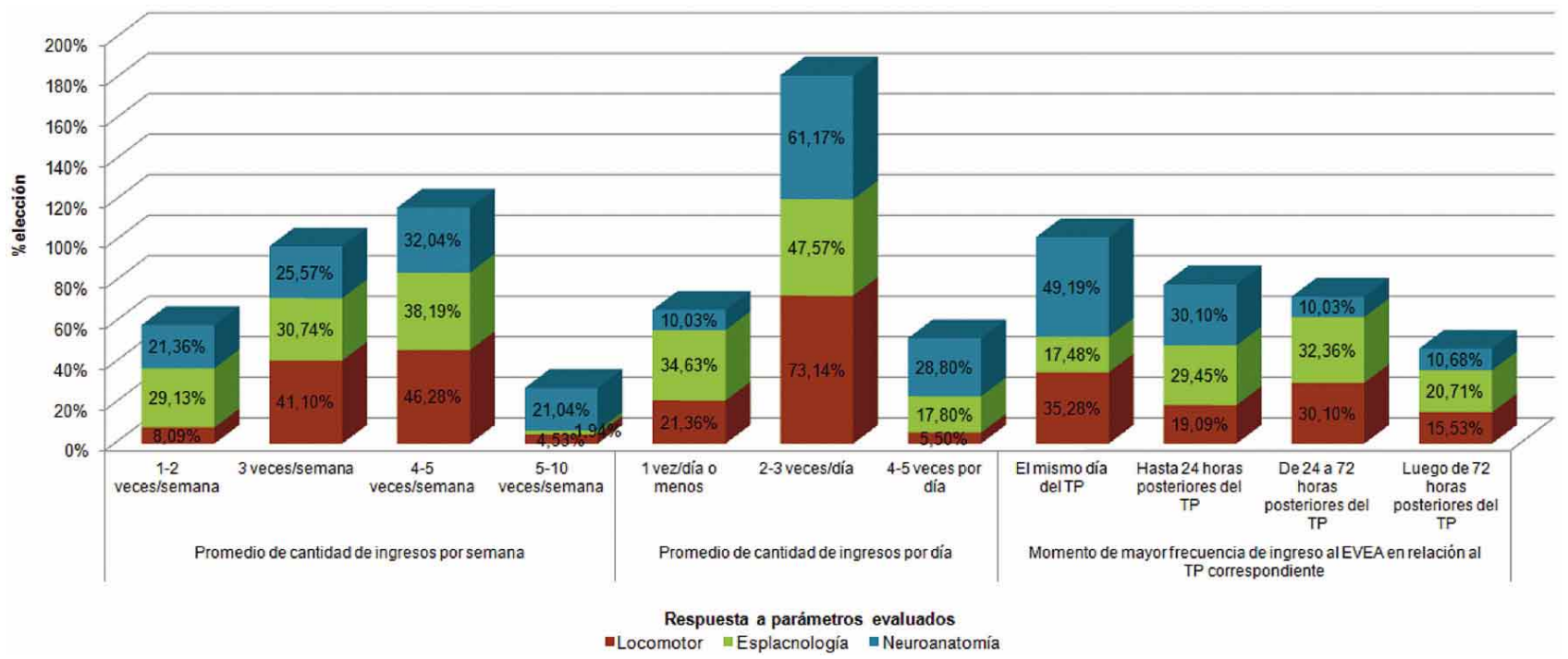

Fig. 2. Porcentaje de respuestas a 3 parámetros evaluados sobre datos de uso del EVEA en función del área de la materia. 
dió en las 3 áreas, pero en locomotor y esplacnología fue seguida por $1 \mathrm{vez}$ por día o menos y en neuroanatomía por $4 / 5$ veces por día. Al evaluar los resultados de rangos de frecuencia de ingreso al espacio virtual se registraron comportamientos heterogéneos en las distintas áreas. En locomotor prevaleció el ingreso el mismo día del TP seguido por ingresos a las 24 a 72 horas luego del mismo; en esplacnología registramos los mayores ingresos entre 24 y 72 horas luego del TP seguidos por los ingresos hasta 24 horas luego del TP (y no en el mismo día); mientras que en neuroanatomía el momento de mayor frecuencia fue el mismo día del TP seguido por ingresos hasta 24 horas posteriores al TP (Fig. 2).

Los mayores porcentajes de alumnos según en rango de tiempos de permanencia en el EVEA registrados en función del área de la materia fueron: en locomotor entre 20 y $40 \mathrm{mi}-$ nutos; esplacnología más de 120 minutos; y en neuroanatomía entre 40 y 60 minutos. Al evaluar la banda horaria en de ingreso al EVEA el horario nocturno entre las 20 y 24 horas fue el más prevalente en las 3 áreas. Tanto en locomotor como en neuroanatomía se objetivó que los días de semana era el momento de mayores ingresos, mientras que en esplacnología el ingreso de los alumnos fue indistinto en relación con el momento de la semana (Fig. 3).

Al evaluar la adherencia subjetiva con el espacio virtual en la modalidad de recurso instruccional todos los parámetros presentaron una alta respuesta positiva por parte de los alumnos. Los que tuvieron mayor aceptación positiva (>90\%) se relacionaron con la introducción de ejercicios de imagenología aplicada al estudio de la anatomía; dichos parámetros más valorados destacaban que los casos de imagenología con reconstrucciones $3 \mathrm{D}$ permitieron un me-

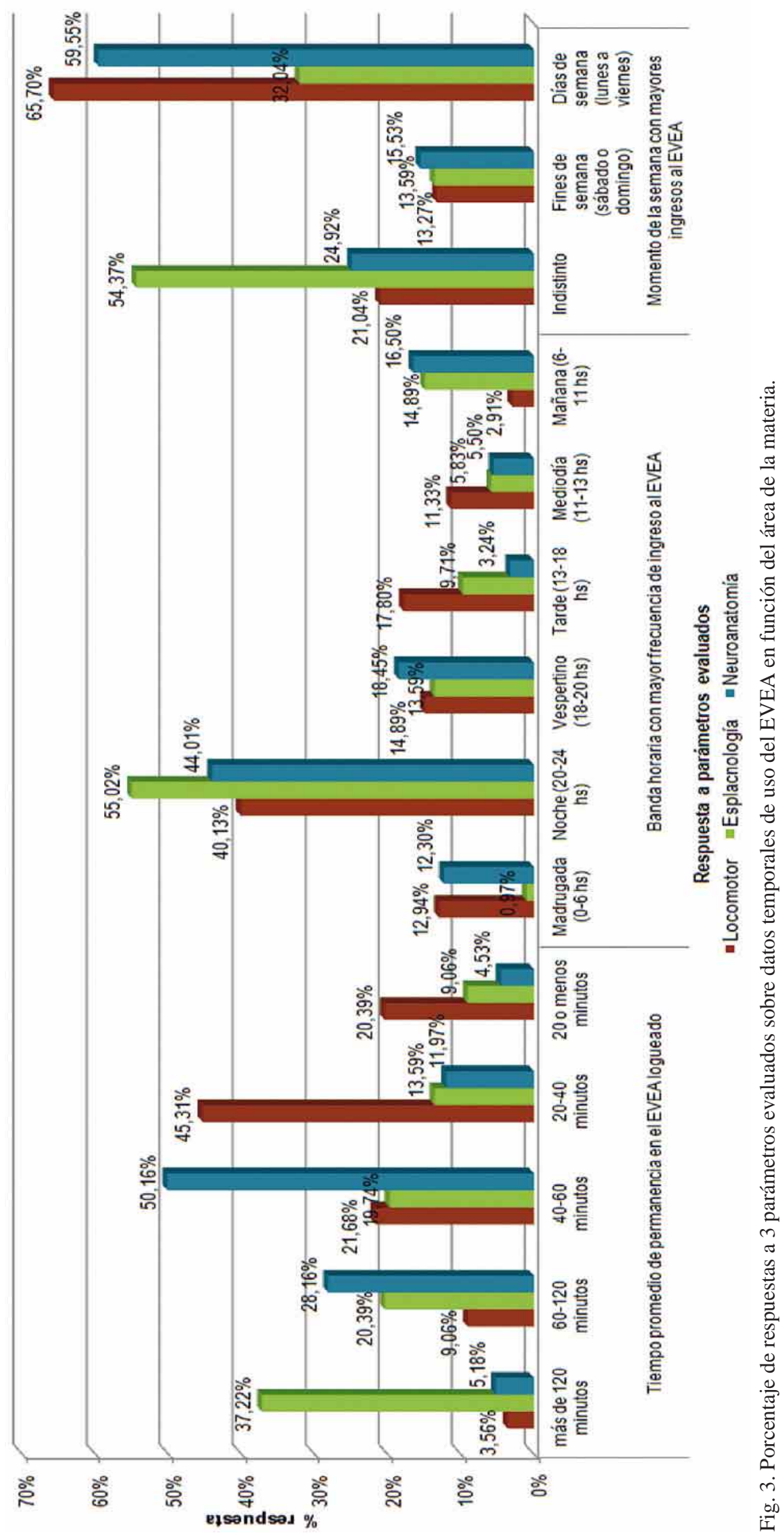


jor manejo con el preparado cadavérico, que el conocimiento de la anatomía normal sirvió para comprender los estudios por imágenes y que los correlatos de imágenes estructurales con preparados anatómicos permitieron un mejor manejo con el preparado cadavérico. Al evaluar los parámetros según área, en locomotor y neuroanatomía los alumnos/usuarios destacaron que el conocimiento de la anatomía normal les sirvió para comprender los estudios por imágenes mientras que en esplacnología recalcaron que los correlatos de imágenes estructurales con preparados anatómicos permitieron mejor manejo con el preparado cadavérico. En locomotor y esplacnología el parámetro con menor respuesta positiva estuvo relacionado a la facilidad de resolver los TP por cuestiones tecnológicas, en neuroanatomía la utilidad de los casos clínico-quirúrgicos para la comprensión de la anatomía normal (Figs. 4-6).

En relación con la gestión de los contenidos en el EVEA, determinamos que las actividades del área de neuroanatomía fueron las que registraron mayor cantidad de veces de realización; las actividades basadas en casos clínico-quirúrgicos presentaron un promedio mayor de realización en relación con las clínico-imagenológicas. Al evaluar el promedio de veces utilizadas para aprobar las actividades, se halló que los casos clínico-quirúrgicos del área de neuroanatomía fueron las que más realizadas, seguidas por

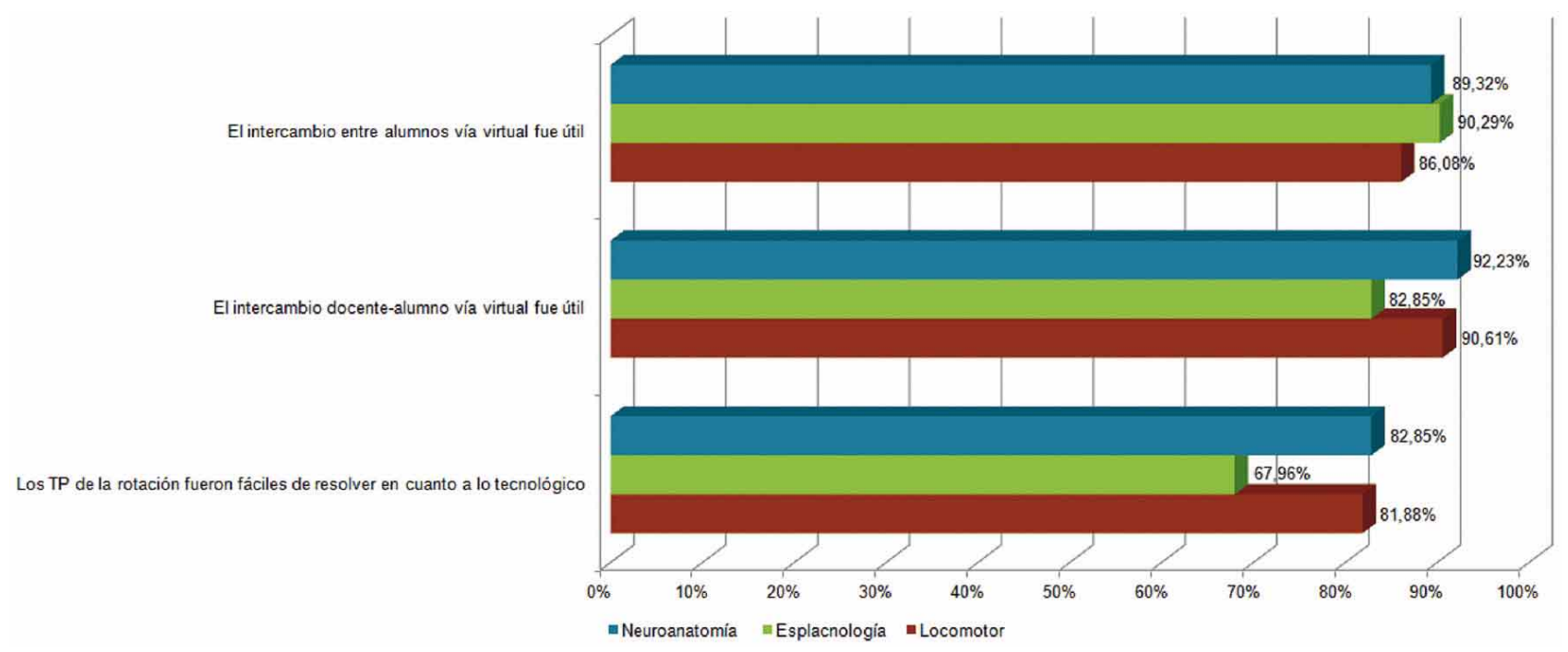

Fig. 4. Porcentaje de respuestas sobre 3 preguntas relacionadas con la adherencia subjetiva de los alumnos en función del área de la materia.

El conocimiento de la Anatomía Normal me sirvió para comprender los estudios de imágenes

El material y los ejercicios de la rotación tuvieron correlación con los conceptos de las clases teóricas

El material y los ejercicios de la rotación tuvieron correlación con los conceptos de las clases prácticas con cadáveres

Los ejercicios y el material adjunto fueron útiles para complementar los TP de la rotación

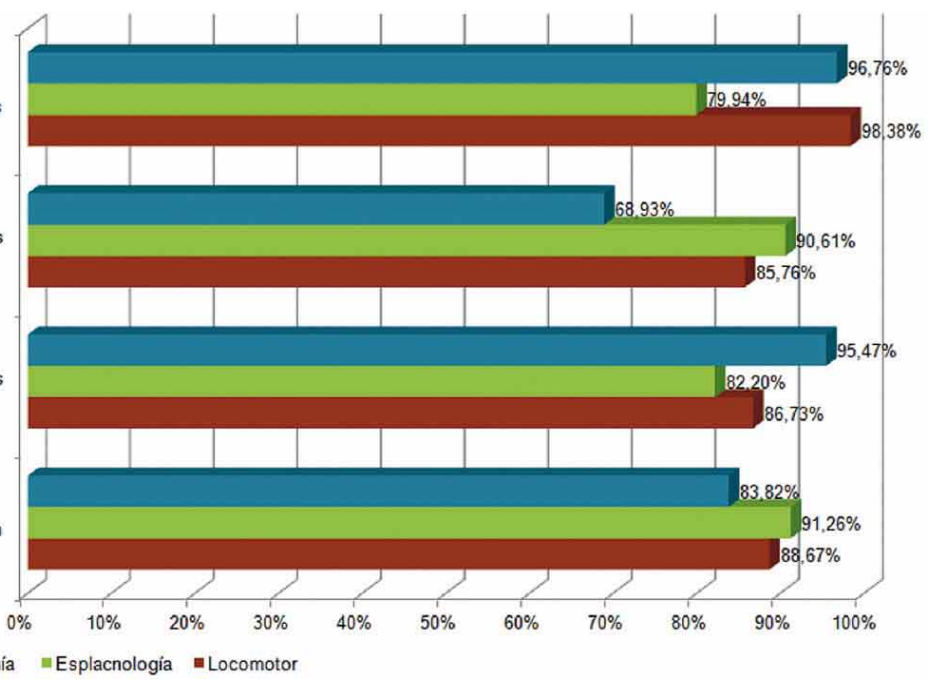

Fig. 5. Porcentaje de respuestas sobre 4 preguntas relacionadas con la adherencia subjetiva de los alumnos en función del área de la materia. 
Los casos de imagenología con reconstrucciones en 3D permitieron mejor manejo con el preparado cadavérico

Los correlatos de imágenes estructurales con preparados anatómicos permitieron mejor manejo con el preparado cadavérico

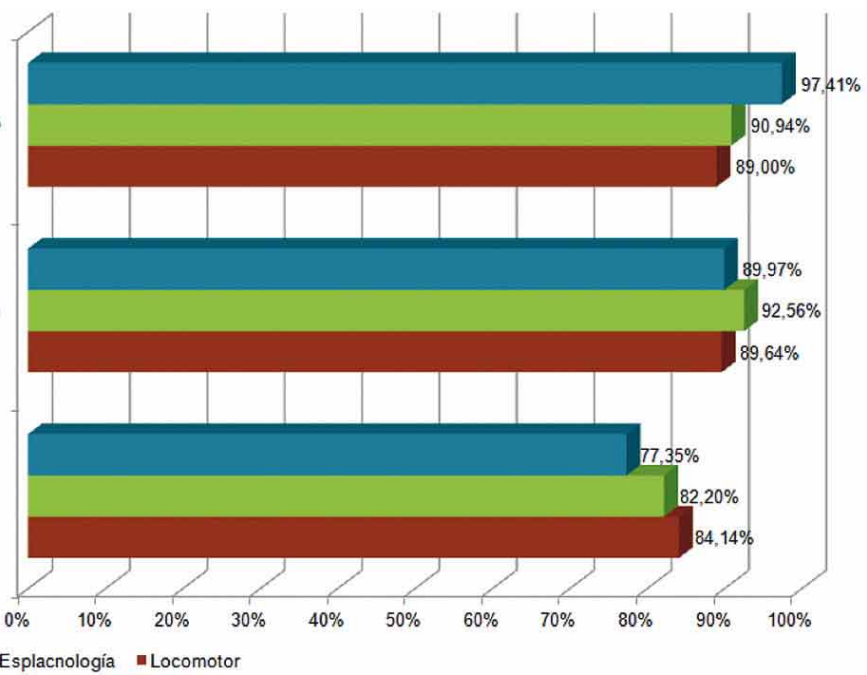

Fig. 6. Porcentaje de respuestas sobre 3 preguntas relacionadas con la adherencia subjetiva de los alumnos en función del área de la materia.

los casos clínico-imagenológicos de esa rotación y luego los casos clínico-quirúrgicos de esplacnología (Fig. 7). Al evaluar el promedio de veces en que los usuarios aprobaron las actividades realizadas, registramos que la mayor prevalencia fue en casos clínico-quirúrgicos de neuroanatomía, seguidos por los casos clínico-imagenológicos de esplacnología; la mayor prevalencia de cantidad de veces desaprobadas se halló en las actividades de casos clínicoimagenológicos de neuroanatomía y clínico-quirúrgicos de esplacnología. Es dable destacar que en los casos clínicoquirúrgicos de esplacnología y de neuroanatomía se hallaron alumnos que desaprobaron 2 o más veces la misma actividad de forma repetida (Fig. 8).

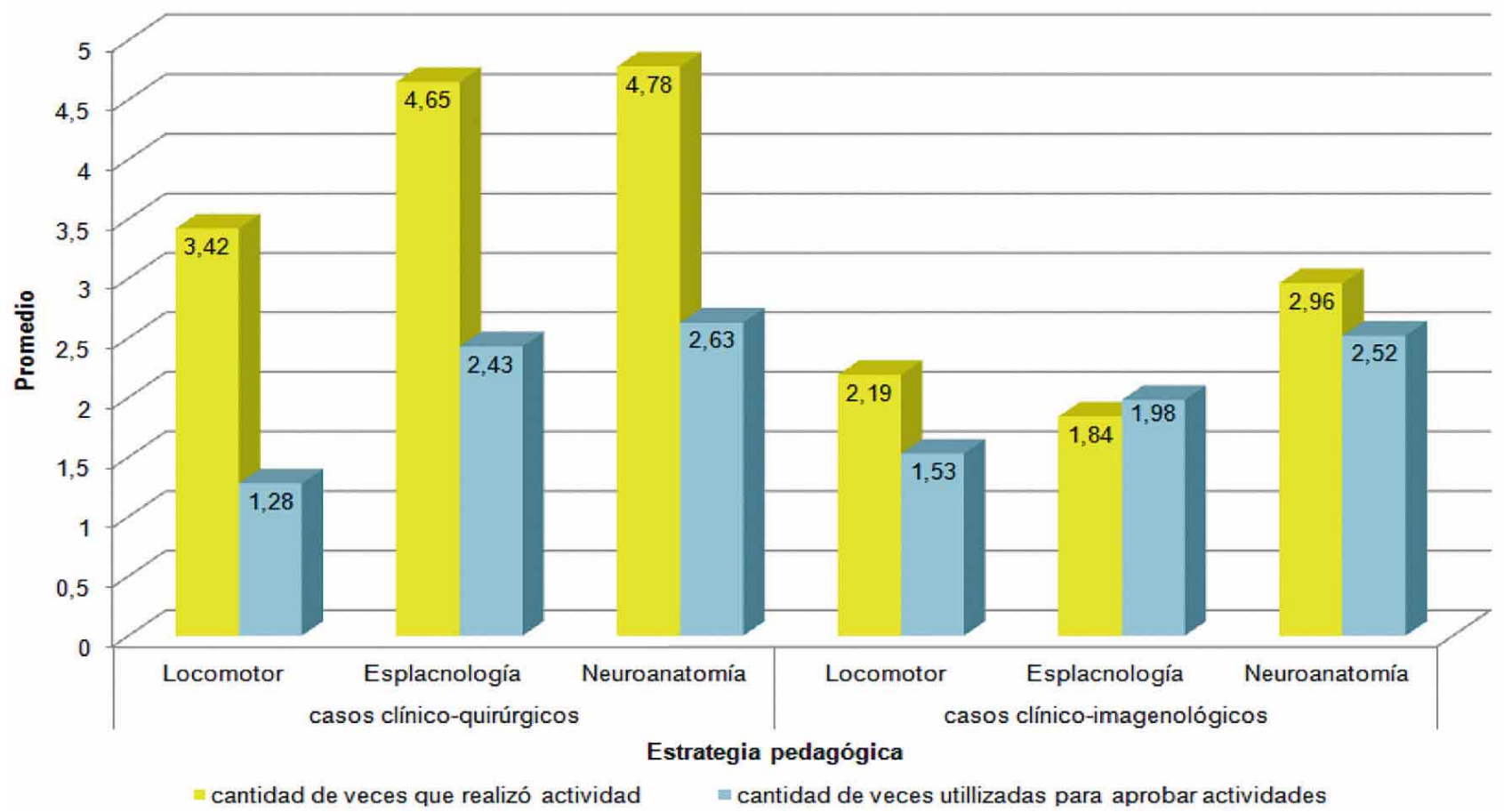

Fig. 7. Promedio de veces utilizadas para realización de actividades en el EVEA según estrategia pedagógica de la mismas. 


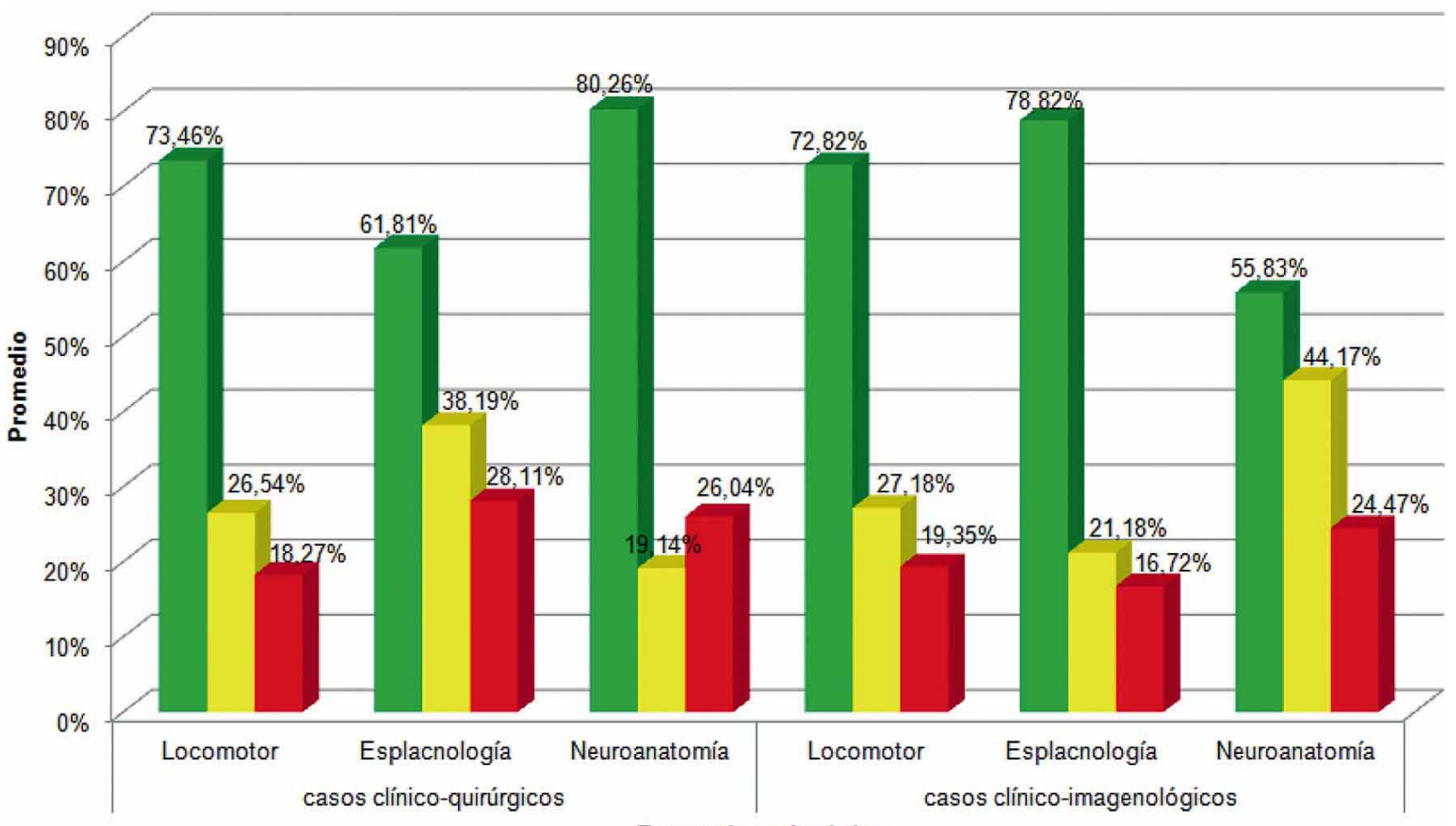

Estrategia pedagógica

- veces aprobadas $\llbracket$ veces reprobadas alumnos que desaprobaron repetidamente 2 o más veces la misma actividad

Fig. 8. Promedio de intentos utilizados y sus resultados en las actividades del EVEA según estrategia pedagógica de la mismas.

Por gráfico de correlación objetivamos que la cantidad de horas de trabajo semanales se relacionaban y estaban implicadas con las características de tiempo de logueo máximo medido en minutos $\left(\mathrm{r}^{2}=0,836\right)$ y los ingresos por semana $\left(r^{2}=0,819\right)$ (Fig. 9). La jornada laboral medida en horas se asoció con 2 parámetros de uso del recurso pedagógico: las veces por semana $\left(\mathrm{r}^{2}=0,857\right)$ y las veces por día $\left(\mathrm{r}^{2}=0,831\right)$ de uso del EVEA (Fig. 10). Por último, el resultado satisfactorio en el examen no se asoció significativamente ni se implicó con los parámetros de uso del recurso (ingresos por semana, tiempo de logueo máximo en minutos) (Fig. 11).

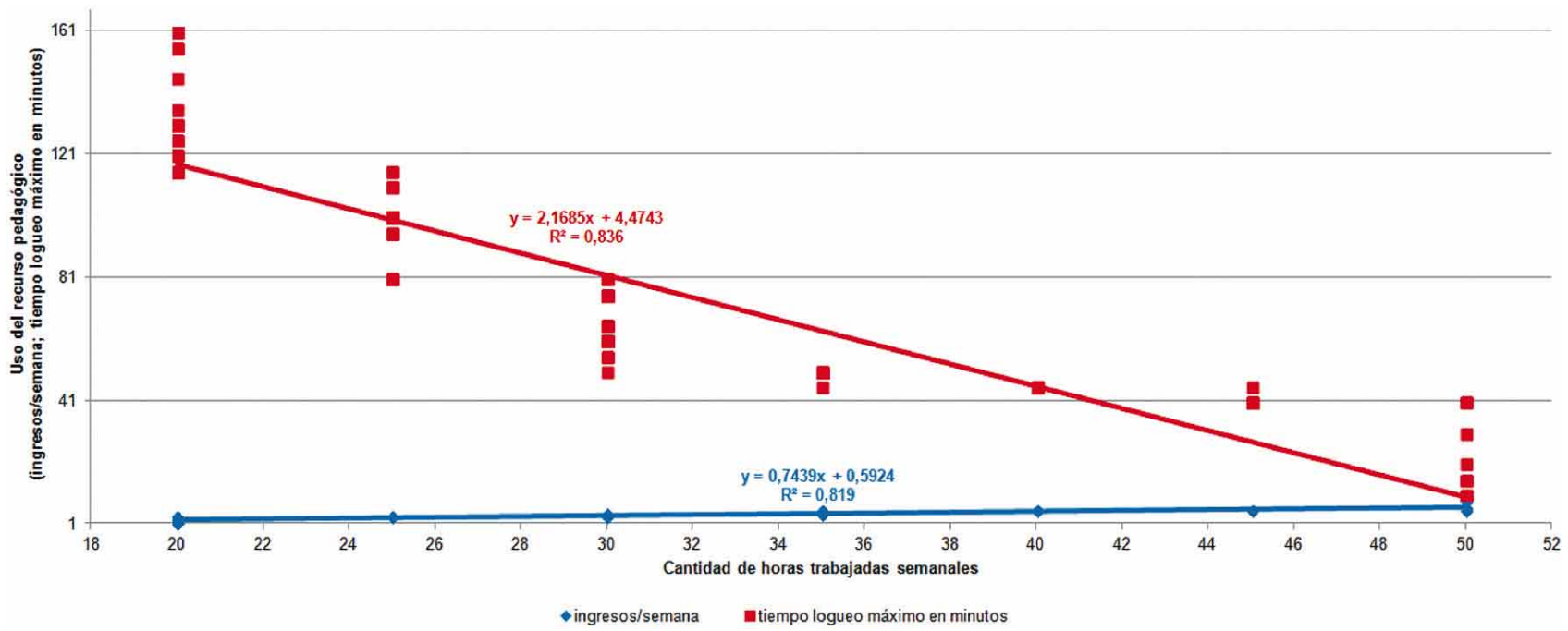

Fig. 9. Correlación entre la cantidad de horas trabajadas semanales y parámetros de uso del EVEA. 


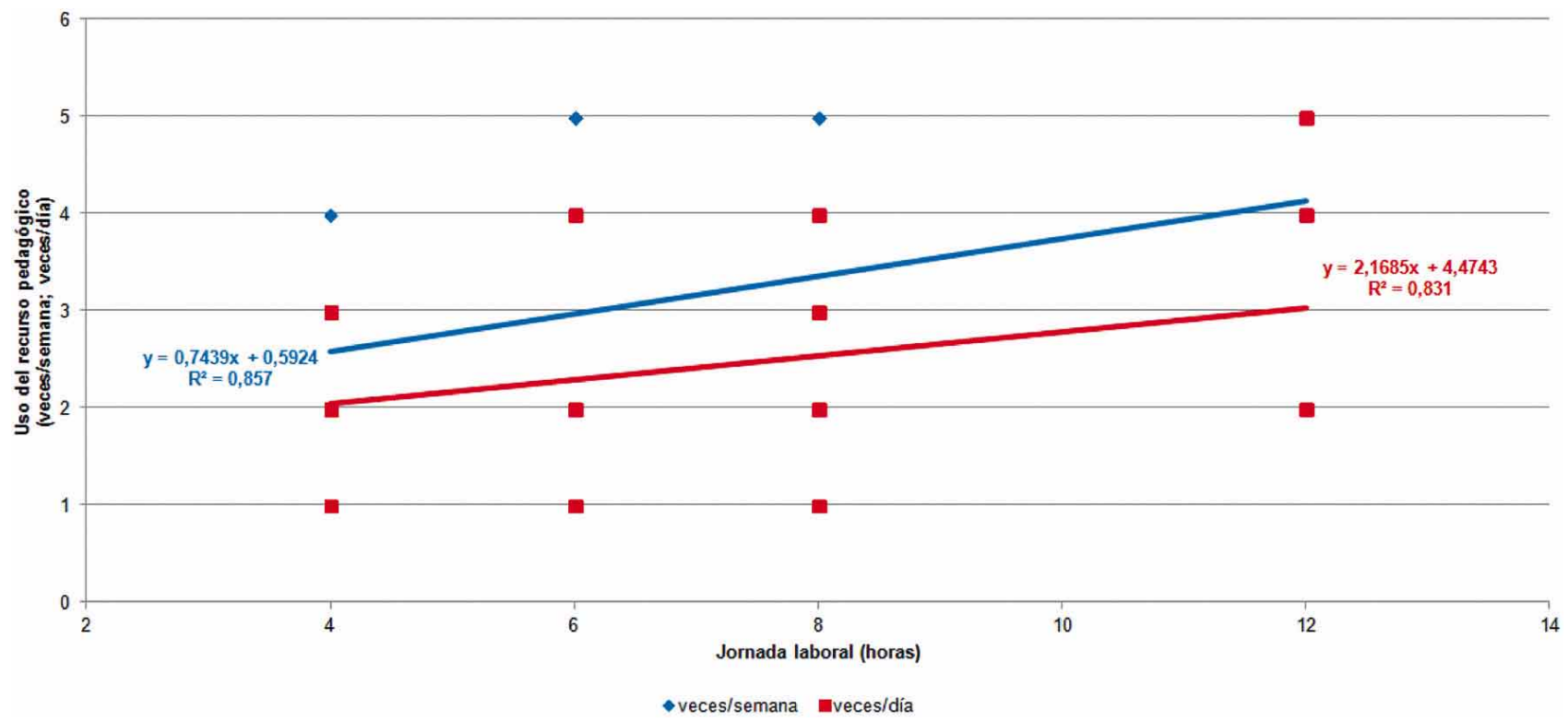

Fig. 10. Correlación entre las horas de jornada laboral y parámetros de uso del EVEA.

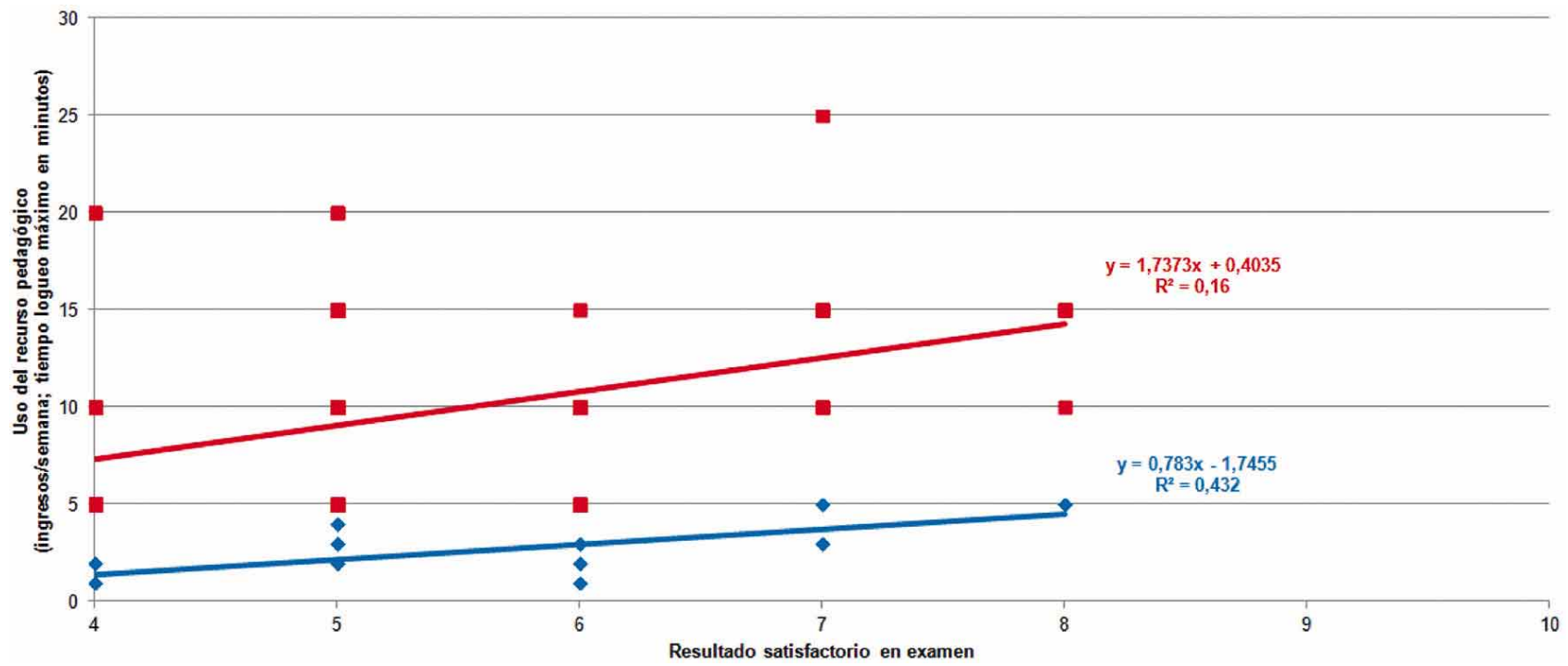

Fig. 11. Correlación entre el resultado satisfactorio obtenido en el examen y los parámetros de uso del EVEA.

\section{DISCUSIÓN}

La enseñanza de la anatomía ha sido llevada a cabo con pocas innovaciones pedagógicas y el objetivo de alcanzar un aprendizaje significativo en los educandos tuvo la característica de ser alumno-dependiente en la gran mayoría de los casos. Las metodologías clásicas de enseñanza y el contexto institucional socio-educativo en el cual se desarrollaron, sin obviar el subjetivo del educando, determinó una heterogeneidad de aprendizaje alcanzado en el cual la estructura cognitiva del receptor fue determinante del conocimiento adquirido.
Actualmente, bajo un paradigma post-moderno de las ciencias de la educación, el alumno tiene un rol activo y dinámico en el proceso de enseñanza y aprendizaje. El alumno debe "construir" su aprendizaje y para ello valerse de todos los medios que le sean más eficaces atento a su estructura cognitiva. Este nuevo rol implica que la búsqueda y adquisición del mismo debe no ser estructurado, pero también fusiona la enseñanza y el aprendizaje como una díada indisoluble en un proceso bidireccional que no puede separarse. 
Dado que el aprendizaje surge de la interacción entre el sujeto (en tanto educando biopsicosocial con una estructura cognitiva individual) con el medio, su subjetividad y especificidad cognitiva determina que procese de diversas formas y con distinto impacto las información que reciba por todos los sentidos. El poder utilizar distintas vías sensoriales de forma sincrónica y análoga de un tema aumenta la capacidad de aprendizaje del mismo. El aprendizaje basado en la teoría constructivista permite una activa e individual forma de aprendizaje y reconoce distintos estilos cognitivos en los educandos los cuales deben ser estimulados por el docente. El docente tiene un rol de facilitador en la construcción del conocimiento por parte del estudiante para lograr un aprendizaje significativo en un contexto social y participativo.

En el campo de la medicina, las TICs han ingresado y se han posicionado en muchas áreas, no sólo en la docente o de actualización sino también en la investigación, difusión de materiales y hasta para temas relacionados con lo asistencial como procedimientos de diagnóstico o ateneos internacionales. Pero el desarrollo tecnológico no ha sido paralelo al desarrollo pedagógico ni a un introducción en los claustros de medicina. En el caso de los EVEA, existen reportes aislados de su uso en materia de ciencias básicas (como anatomía, histología, anatomía patológica, entre otras) bajo la forma de recurso complementario o reforzador de una forma de enseñanza clásica. En muchos otros casos son utilizados al estilo de carteleras virtuales asincrónicas para difusión de noticias o archivos pero sin interacción entre los usuarios ni con los docentes.

La mayoría de los reportes (Avila et al.; Buelta Carrillo et al.; Inzunza et al.; Inzunza \& Bravo, 1999, 2002) manifiestó una alta aceptación y adhesión de los alumnos con este recurso, pero no se evaluaron de forma consistente la ventajas que se obtienen con este recurso pedagógico ni el tipo o estrategias de aprendizaje que generan, fomentan o amplían (Wiecha \& Barrie).

La masiva y rápida introducción de múltiples tecnologías para la información y la comunicación y su amplia diversificación entre los distintos grupos sociales determinaron que el recurso tecnológico se posicionase como un elemento más en la cotidianeidad. De esta forma pasaron de ser utilitarios para cuestiones administrativo-laborales a ser medios para la conectividad social, mas actualmente generan espacios de aprendizaje con múltiples posibilidades. Las posibilidades que brindan los EVEA, tanto de forma sincrónica como asincrónica, permiten la enseñanza en un contexto social y participativo que obliga a los docentes a realizar un armado de los contenidos y gestionarlos para alcanzar su maximización (Lara \& Duart). Las herramientas de gestión del EVEA son dinámicas en tanto los usuarios/educandos "crean" sus nuevos escenarios y posibilidades. De esta forma se modifica la educación, su contexto y su finalidad pudiendo concretarse la misma durante las tareas laborales o conjuntamente con momentos de ocio. Pero la gestión de los contenidos no debe reducirse al armado y puesta en funcionamiento sino también a poder realizar un seguimiento del proceso enseñanza-aprendizaje, evaluación de los objetivos de enseñanza alcanzados por los usuarios y evaluación de las técnicas y estrategias pedagógicas aplicadas a los contenidos en pró de lograr la mayor eficiencia.

\section{CONCLUSIONES}

La adhesión al uso del espacio virtual como recurso instruccional complementario a los trabajos prácticos y clases teóricas de Anatomía para la carrera de Medicina fue alta en todas las áreas que componen la materia. Esta adhesión presentó especificidades según las áreas de la materia, principalmente en el contexto físico de desarrollo de las actividades, forma de acceso al espacio virtual, bandas horarias de ingreso y estrategias pedagógicas de las actividades, las cuales estuvieron relacionadas con la heterogeneidad de los usuarios y del objeto de estudio. Los parámetros de gestión de los contenidos en el espacio virtual evidenciaron diferencias sustanciales entre cada área y demostraron diferencias de rendimiento relacionadas con las estrategias pedagógicas de las actividades implementadas.

ALGIERI, R. D.; TORNESE, E. B.; MAZZOGLIO Y NABAR, M. J.; DOGLIOTTI, C. G.; GAZZOTTI, A.; JIMÉNEZ VILLARRUEL, H. N. \& REY, L. EVEA in Anatomy: accession and content management by area. Int. J. Morphol., 32(3):850-859, 2014.

SUMMARY: Preliminary studies and qualitative and quantitative benefits ratified the use of virtual environments for teaching and learning (VLE) in both anatomy and cognitive performance and socio-pedagogical aspects. The objective of the present study was to assess adherence, content management and use of undergraduate students as a EVEA areas that make up matter and user characteristics. An observational, retrospective and crosssectional study on usage characteristics and content management (administrative and academic) activities of clinical anatomy (anatomical-surgical and anatomical-imaging) through a VLE in 309 of the 2011 students. Activities were divided by subject areas and applied statistical parameters (descriptive and inferential) results. The work complied with the ethical and legal regulations. The majority entered EVEA from public spaces with free and mobile computers. The 3 areas had an average of 4/5 weekly earnings, the difference being that during splanchnology users were 
not admitted on the day of cadaveric practical work and the longer residence time activities with clinical and surgical cases had higher completion attempts with worse outcomes. The working hours were correlated with parameters of use (quantity and time of logging). In conclusion adherence to the virtual space provided according to specific subject areas that were related to labor characteristics of users and affected the yield obtained in the activities according to the teaching strategy of them.

KEY WORDS: Anatomy; Pedagogy; Virtual space for teaching and learning; Accession; Management.

\section{REFERENCIAS BIBLIOGRÁFICAS}

Algieri, R. D.; Mazzoglio y Nabar, M. J. \& Castro Barros, F. A. Espacios virtuales de enseñanza y aprendizaje (EVEA) en esplacnología: especificidades pedagógicas en su enseñanza topográfica. Int. J. Morphol., 30(3):908-15, 2012a.

Algieri, R. D.; Mazzoglio y Nabar, M. J.; Dogliotti, C. G. \& Gazzotti, A. TICs Aplicadas a la enseñanza del aparato digestivo. Int. J. Morphol., 27(4):1261-8, 2009.

Algieri, R. D.; Mazzoglio y Nabar, M. J.; Dogliotti, C. G.; Gazzotti, A.; Jiménez Villerruel, H. N.; Rey, L. \& Gómez, A. Especificidades pedagógicas en la utilización de un EVEA para la enseñanza del aparato locomotor. Rev. Arg. Anat. Onl., 3(4):109-15, 2012b.

Algieri, R. D.; Mazzoglio y Nabar, M. J.; Dogliotti, C. G.; Gazzotti, A.; Rey, L. \& Gómez, A. Rendimiento en casos clínicoimagenológicos a través del espacio virtual. Rev. Hosp. Aeronáut. Cent., 7(2):111-7, 2012c.

Algieri, R. D.; Mazzoglio y Nabar, M. J.; Dogliotti, C. G.; Rey, L.; Gómez, A. \& Tornese, E. B. Espacio virtual de enseñanza y aprendizaje aplicado en la enseñanza del tórax: adherencia y utilidad didáctica. Rev. Hosp. Aeronáut. Cent., 7(2):37-9, 2011.

Avila, R. E.; Samar, M. E. \& Peñaloza Segura, F. Descripción y Evaluación de una página Web como Apoyo Virtual del Curso Presencial 2003 de Biología, Histología y Embriología en la carrera de Medicina. Primer Congreso Virtual Lationamericano de Educación a Distancia. LatinEduca2004, 2004. Disponible en: www.ateneonline.net/datos/09_01_Avila_Rodolfo_y_ otros.pdf

Buelta Carrillo, L.; Fernández Fernández, F.; Garijo Ayensa, F. \& Val Bernal, F. Docencia virtual de anatomía patológica. Rev. Esp. Patol., 36(2):139-48, 2003.

Inzunza, O. \& Bravo, H. Animación computacional de fotografías: un real aporte al aprendizaje práctico de anatomía humana. Rev. Chil. Anat., 20(2):151-7, 2002.

Inzunza, O. \& Bravo, H. Impacto de dos programas computacionales de anatomia humana en el rendimiento del conocimiento practico de los alumnos. Rev. Chil. Anat., 17(2):205-9,1999.

Inzunza, O.; Bravo, H.; García Huidobro, C.; Contreras, P.; Riquelme, S. \& Valenzuela, A. Software docente para la enseñanza en anatomía. Rev. Chil. Anat., 11:81, 1993.

Lara, P. \& Duart, J. M. Gestión de contenidos en el e-learning: acceso y uso de objetos de información como recurso estratégico. Rev. Univ. Soc. Conoc., 2(2):6-16, 2005.

Popescu, B. M. \& Navarro, V. Comparación del aprendizaje en internet con la clase convencional en estudiantes de medicina, en Argentina. Educ. Méd., 8(4):28-31, 2005.

Tornese, E. B.; Dogliotti, C. G.; Mazzoglio y Nabar, M. J.; Algieri, R. D.; Gazzotti, A.; Jiménez Villarruel, H. N.; Rey, L. \& Gómez, A. Entorno virtual de enseñanza y aprendizaje aplicado como recurso instruccional complementario en la enseñanza de neuroanatomía:aspectos poblacionales, didácticos y psicopedagógicos. Int. J. Morphol., 29(4):1130-5, 2011.

Tornese, E. B.; Mazzoglio y Nabar, M. J.; Algieri, R. D.; Dogliotti, C. G. \& Gazzotti, A. mEVEA: ¿Espacios de enseñanza sin límites? Rev. Docencia Univ., 13(1):57-67, 2012.

Wiecha, J. M.; Gramling, R.; Joachim, P. \& Vanderschmidt, H. Collaborative e-learning using streaming video and asynchronous discussion boards to teach the cognitive foundation of medical interviewing: a case study. J. Med. Internet Res., 5(2):e13, 2003.

Dirección para Correspondencia:

Martín Javier Mazzoglio y Nabar

Médico, Especialista en Psiquiatría

Jefe de Trabajos Prácticos de Anatomía

III Cátedra de Anatomía.

Docente Auxiliar de Farmacología y Psiquiatría

Facultad de Medicina

Universidad de Buenos Aires

Buenos Aires

ARGENTINA

Email: mazzoglioynabar@hotmail.com

Recibido: 16-12-2013

Aceptado: 19-05-2014 